\title{
Enfermería: evolución, arte, disciplina, ciencia y profesión
}

\author{
Nursing: evolution, art, discipline, science and profession
}

Enfermagem: Evolução, arte, disciplina, ciência e profissão

\author{
Marlin Angélica Guía-Yanes \\ mguia28@hotmail.com
}

Recibido septiembre 2018 / Revisión octubre 2018 / Aceptado 1 de enero 2019

\begin{abstract}
RESUMEN
Introducción: enfermería se remonta a tiempos inmemorables, considerada como el arte y la ocupación del cuidado a los pacientes. Es una disciplina que se caracteriza por ser independiente a las otras ramas de la salud con las cuales trabaja de forma integrada, pasando a convertirse, en el eslabón primordial en toda institución sanitaria. Objetivo: Este artículo pretende analizar la evolución y cambios suscitados en la profesión de enfermería, considerando los aportes realizados por Florence Nightingale como precursora principal del cambio de la enfermería hacia la formación como profesión. Metodología: Revisión de literatura virtual, portales reconocidos (Scielo, Dialnet, servicio.bc.uc.edu.ve, repositorios institucionales) Se revisaron artículos originales y de revisión, en los idiomas inglés y español según los descriptores asociados (enfermería; historia de la enfermería; atención de enfermería). Se excluyeron artículos y materiales que no incorporaban los descriptores. Conclusión: La carrera de enfermería posee características únicas dentro del campo de la medicina, ya que requiere condiciones y formación precisa para desempeñarse como una ocupación, arte, disciplina y ciencia.
\end{abstract}

Palabras clave: enfermería; historia de la enfermería; atención de enfermería

\section{ABSTRACT}

Introduction: The profession of nursing dates back to immemorial times, regarded as the art and occupation of patient care. It is a discipline that is characterized by being independent of the other branches of health with which it works in an integrated way, becoming, and the primary link in any health institution. Objective: This article aims to analyze the evolution and changes that have arisen in the nursing profession since its inception, considering the contributions made by Florence Nightingale as the main precursor of the change of nursing towards training as Profession. Methodology: A descriptive, simple and precise review was carried out in order to provide useful concepts in this area of constant evolution, highlighting the importance of its teaching and its general progress as a profession. Conclusion: Nursing career has unique characteristics within the field of medicine, as it requires precise conditions and training to perform as an occupation, art, discipline and science.

Key words: nursing; nursing history; nursing care
MG: Universidad Central de Venezuela, Venezuela. 
MG: Universidad Central de Venezuela, Venezuela.

\section{RESUMO}

Introdução: A profissão de enfermagem remonta aos tempos imemoriais, considerados como a arte e ocupação do atendimento ao paciente. É uma disciplina que se caracteriza por ser independente dos demais ramos da saúde com os quais trabalha de forma integrada, tornando-se, o elo primário em qualquer instituição de saúde. Objetivo: Este artigo visa analisar a evolução e as mudanças que surgiram na profissão de enfermagem desde sua criação, considerando as contribuições feitas por Florence Nightingale como a principal precursora da mudança de enfermagem para a formação como Profissão. Metodologia: Uma revisão descritiva, simples e precisa foi realizada com o objetivo de proporcionar conceitos úteis nessa área de evolução constante, destacando a importância de seu ensino e seu progresso geral como profissão. Conclusão: A carreira de enfermagem tem características únicas no campo da medicina, pois exige condições precisas e treinamento para atuar como ocupação, arte, disciplina e ciência.

Palavras-chave: enfermagem; história da enfermagem; cuidados de enfermagem

\section{INTRODUCCIÓN}

$\mathrm{E}$ l cuidado de la salud en las personas se remonta a tiempos remotos, y es reseñado en el libro más antiguo: "La Biblia", donde se puntualiza acerca del cuidado realizado a quienes padecían lepra o a las madres cuidando a sus niños. El cuidado ha evolucionado de ser una práctica doméstica, a ser considerado un arte, un oficio, una disciplina hasta llegar a convertirse una profesión con un cuerpo científico propio.

En principio al no poseer una fundamentación teórica y realizar los cuidados de manera empírica con la lógica que dictan las necesidades de la persona fue estimada como una ocupación, a la cual se integraron las habilidades, destrezas y esmero en su realización que hicieron que se considerara como disciplina, posteriormente con el aporte fundamental de Florence Nightingale se logra un cambio total en la concepción de enfermería (1), y se hace necesaria la realización de estudios superiores en universidades y ser incluida como una profesión.

Nightingale se ocupó de formar a las enfermeras para ello implemento un programa de estudios que buscaba profesionalizarlas (1). Su contribución como docente, fue de gran importancia, con sus innovaciones basadas en la ciencia. Expresó su firme convicción de que el conocimiento de la enfermería -no sólo su práctica- era intrínsecamente distinto del de la ciencia médica. En este marco, definió la función propia y distinta de la enfermera (colocar al paciente en las mejores condiciones para que la naturaleza actúe sobre él) y defendió la idea de que esta profesión se basaba en el conocimiento de las personas y su entorno (punto de partida diferente al utilizado por los médicos para su ejercicio profesional).

El término "profesión" utilizado desde hace más de 4 siglos, significa "vocación" aprendida, la profesión se diferencia de un oficio en que aquélla tiene una base científica de donde procede el servicio que ofrece a la sociedad, este cimiento teórico se debe revisar y acrecentar a medida que se originan nuevos descubrimientos.

Posterior a Nigthingale los teóricos de enfermería se han dedicado a investigar acerca de la elaboración de un marco conceptual, teorías y filosofía de los cuidados, que son el fundamento para 
llegar a un orden nuevo, es decir, a un nuevo conocimiento. Entre las teorizantes con importantes aportes se puede mencionar a Martha Rogers según sus premisas la enfermería despliega un rol de "ayudar al paciente en la reorientación de los acontecimientos vitales hacia patrones que le conduzcan a una salud óptima". Plantea Rogers examinar al ser humano respecto a sí mismo y al entorno, y gestionar los cuidados enfermeros en función de los cambios del entorno que son los que provocan cambios en el ser humano (2).

Dorothea Orem, otra teorizante de enfermería, se centró en: las necesidades del individuo, por las actividades del autocuidado, su prestación y tratamiento de forma continuada para mantener la vida y la salud; recuperarse de enfermedades o lesiones y enfrentarse a sus efectos (3). Virginia Henderson, planteó un modelo donde del papel fundamental de enfermería es ayudar al individuo, sano o enfermo, a conservar o recuperar su salud (o bien asistirlo en los últimos momentos de su vida) para cumplir aquellas necesidades que realizaría por sí mismo si tuviera la fuerza, voluntad los conocimientos necesarios $(4,5)$. Johnson según su modelo la enfermería considera al individuo como una serie de partes interdependientes, que funcionan como un todo integrado; estas ideas fueron adaptadas de la Teoría de Sistemas.

Lidia Hall por su parte señala que el único campo exclusivo de la Enfermería es el círculo del cuidado que comprende el aspecto corporal personal; mientras que los círculos del núcleo y la curación los comparte con profesionales de otras disciplinas (4). Imogene King preciso que enfermería es una profesión de servicio, que satisface a una necesidad social. Supone planificar, aplicar y evaluar los cuidados de enfermería (6). Entre otras que han hecho su aporte para lograr el desarrollo disciplinar de la profesión y establecer un marco teórico conceptual sobre el cual están basados los principios y objetivos que la orienten de la profesión. Todas desde Nigthingale coinciden en el cuidado como centro de la profesión y establecen un marco teórico conceptual.

El cambio de perfil de la enfermería tradicional en su antigua preparación a un enfermero profesional hoy día, ha requerido una reinvención de su comportamiento en su visión del mundo, de las necesidades en materia de salud, así como de un enfoque complejo de cuidado integral a los pacientes, con la puesta en práctica de acciones independientes e interdependientes, desde un marco integral apoyado en criterios y teorías.

Los procesos a través de los cuales ha evolucionado la enfermería, han permitido incrementar el número de personal calificado en cuanto a conocimientos científicos y prácticos, que permiten su desarrollo cada día con aportes teóricos en beneficio del cuidado de calidad a la población.

Adaptándose a los cambios y exigencias de la sociedad y la tecnología, se realiza también cambios en la enfermería, pasando de ocupación a profesión a través de la profesionalización con los estudios de nivel universitario cursados en un instituto de educación superior.

Este artículo pretende analizar la evolución y cambios suscitados en la profesión de enfermería desde sus inicios cuando se consideraba arte y su evolución a disciplina, ciencia y profesión considerando los aportes realizados por Florence Nightingale como precursora principal del cambio de la enfermería hacia la formación como profesión. 


\section{METODOLOGÍA}

$\mathrm{S}$ realizó una

búsqueda considerando los artículos publicados en los principales sitios web; para la indagación se utilizó el metabuscador (google académico) y bases digitales en portales reconocidos (Scielo, Dialnet, servicio.bc.uc.edu.ve, repositorios institucionales y libros de teorizantes de enfermería) se consideraron aquellas publicaciones y libros originales que permitieron establecer un orden histórico en la evolución de enfermería como profesión basada en un cuerpo de conocimientos científicos que la hacen merecedora de tal consideración, partiendo de sus inicios como ocupación. Se revisaron artículos originales y de revisión en los idiomas inglés y español según los descriptores asociados (enfermería; historia de la enfermería; atención de enfermería). Se excluyeron artículos y materiales que no incorporaban los descriptores.

\section{DISCUSIÓN}

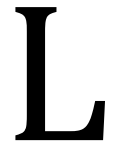
a historia referida a la enfermería, señala a Florence Nightingale como la primera enfermera de ocupación reconocida, nacida en Florencia, Italia y criada en Derbyshire, Inglaterra, proveniente de una familia de alto status social, donde se dedica a estudiar enfermería. En 1850 inició estudios en el Instituto San Vicente de Paúl en Alejandría, Egipto. Posteriormente, estudió en el Instituto para Diaconisas Protestantes de Kaiserswerth, Alemania (7). Continuó su formación como enfermera en Alemania, 1851, en una comunidad religiosa protestante que contaba con un centro hospitalario, donde se preparaban mujeres para brindar cuidados de manera empírica.

Nightingale, jugó un papel importante durante la guerra de Crimea (1854-1856) curando a heridos y enfermos. Después de esta guerra, se dedicó a impartir y mejorar la educación de la enfermería, donde su mayor aporte fue la creación de nuevas instituciones para la formación tanto de médicos militares como enfermeras de hospital (8).

Nightingale desarrolló el primer programa organizado de formación para enfermeras, en 1860 en la llamada Nightingale Training Schoool for Nurses, unida al St. Thomas's Hospital. El propósito de la escuela era capacitar enfermeras para laborar en los hospitales, enfermeras prácticas cuya dedicación fuera la de atender enfermos de escasos recursos económicos, y por otro lado enfermeras desarrollar habilidades para para formar a otras. Los contenidos teóricos eran dictados por médicos y la formación práctica estaba a cargo de una enfermera entrenada (9) Esta formación de enfermeras sirvió posteriormente de modelo a escuelas de enfermería de todo el mundo.

Las artes y Nightingale, fue una mujer luchadora, incansable que durante su vida profesional escribió libros y documentos, hizo aportes relevantes en el área de la salud, sugirió estudios de patología para los médicos. Sus observaciones y anotaciones del proceso saludenfermedad, brindaron un camino claro para las técnicas de asepsia y antisepsia. Se le reconoce como la creadora de los primeros estudios de educación superior para enfermeras a través de la escuela de formación donde egresaron las primeras enfermeras tituladas con estudios superiores, siguiendo luego, con la creación de escuelas en enfermería en diversas partes del mundo (8).

Es considerable la contribución realizada por Nightingale, con sus innovaciones basadas en la ciencia, logrando superar el modelo asistencial tradicional, fundamentado en los buenos sentimientos y en el sectarismo religioso, para sustituirse por una asistencia 
sanitaria científica, que precisaba una rigurosa formación del personal de enfermería (7), sin menoscabo del trato digno que debía recibir cada persona cuidada.

Nightingale contaba con sólidos conocimientos en ciencias y matemáticas, dada la educación impartida por su padre, lo que le conduce a realizar importantes reflexiones en materia de salud y un aporte invaluable a las ciencias en general, como lo fue el método estadístico. Innovó en la recolección, tabulación, interpretación y presentación de gráficas de las estadísticas descriptivas, también desarrolló una fórmula modelo de estadística hospitalaria, para que los sanatorios generaran sus datos (7).

Sin duda, los aportes realizados a la enfermería por Florence Nightingale, son invaluables. La etapa profesional de enfermería se inicia a partir del siglo XIX, con mayor énfasis en el siglo XX. Época que marca cambios, ya que surge una importante reflexión intelectual sobre el quehacer enfermero. Situación que exigió contar con una línea de análisis conceptual, la cual tuvo sus orígenes en el siglo XIX, cuando Nightingale realizó una serie de observaciones que la llevaron a reconocer el valor del cuidado. En 1901, en Nueva Zelanda, se aprobó el Acta de Certificación de Enfermeras, que fue la primera y verdadera regulación en materia de prestación de cuidado (10).

Esta precursora de la enfermería, condujo al estudio y teorización del cuidado humano por parte de muchas otras estudiosas de este campo, entre las que destacan los aportes realizados por Henderson (1948), Fray (1953), Oren, Peplau (1952), quienes además, propiciaron el inicio del proceso de atención de enfermería y realizaron definiciones y teorías basadas en el trabajo de las enfermeras, para darles un campo de acción más científico, basado en observaciones y estudios efectuados, contribuyendo así para los posteriores estudios a nivel superior.

La profesión enfermera en su incesante evolución y atendiendo las demandas de la sociedad se ha transformado en las últimas décadas en la ciencia del cuidado, distanciándose paulatinamente del modelo biomédico que se centra en tratar la enfermedad. Esto se comienzo a trascender con la formación de un cuerpo de conocimientos, la utiliza considerando los conocimientos, principios y valores humanos del método científico aplicado a la práctica del cuidado, la postulación de modelos y teorías que fundamentan el quehacer y la misma profesión y en consecuencia emergiendo la autonomía de los cuidados de enfermería en el objeto central de estudio, que es el cuidado del individuo o comunidad.

En la medida que la enfermera se ha empoderado de los cuidados como su principal propósito, y cuenta con argumentos teóricos, es capaz de perfilar un quehacer autónomo e indelegable. La autonomía y su asociada responsabilidad se evidencian en la legislación de los países que regula el quehacer profesional. La profesión enfermera se ha convertido en una ciencia que abarca en sus planteamientos las necesidades y las respuestas humanas, tal como lo han evidenciado las diferentes teorizantes(5).

Para conseguir el desarrollo disciplinar de la profesión ha sido preciso establecer un marco teórico conceptual sobre el cual están soportados los principios y objetivos de la profesión. Así como adecuar un método científico usando una metodología propia para resolver los problemas competentes a la enfermería. Esto implica la utilización de un método sistemático, el Proceso Enfermero(PE) para proporcionar cuidados centrados en 
la obtención de los resultados preestablecidos y esperados.

El Proceso Enfermero (PE) o Proceso de Atención de Enfermería (PAE) en la práctica diaria como método científico para la gestión y administración de los cuidados ha sido fundamental para el desarrollo de la disciplina enfermera (5) que se define como un método sistemático que permite de forma planificada e intencionada y gestionar cuidados de enfermería de manera individualizada

En la profesión de enfermería con el tiempo, ha contado con personas que han ejercido una influencia de gran valor al realizar diversas teorías que expliquen y definan su naturaleza, objetivos y práctica.

Algunas de las teorías más representativas de la profesión de enfermería son citadas por Marriner et al (11), en su libro Modelos y teorías de enfermería, donde realiza una distinción entre los términos disciplina y profesión. Refiriéndose a la disciplina como una expresión del mundo académico, hace referencia a una rama de la educación, un departamento educativo o un campo del saber. Mientras que el vocablo profesión se aplica más a un área referida al campo de trabajo, basado en la estructura teórica de la ciencia o del saber de una disciplina y las habilidades prácticas necesarias para desarrollarlo (11).

Rogers, define a la enfermería como una ciencia y un arte. Es una ciencia humanística dedicada a promover y mantener la salud y a prevenir la enfermedad, $\mathrm{y}$ asistir $\mathrm{y}$ rehabilitar al individuo enfermo para fortalecer la coherencia e integridad de los seres humanos y para dirigir y redirigir modelos de interacción entre el hombre y su entorno y así lograr el desarrollo del máximo potencial de salud. Es la única ciencia que trata al ser unitariamente y enfoca la meta de la enfermería, en la promoción de la salud y del bienestar (11).
Ernetine Wiedenbach y Virginia Henderson, describen en sus teorías a la enfermería como un arte, ya que es en el cuidado humano y de sus necesidades, donde se desarrolla la práctica. Henderson dentro de sus teorías y opiniones, concedió importancia a la formación de alto nivel, cuando afirmaba que para que una enfermera pueda ejercer como experta y utilizar el planteamiento científico, necesita una educación universitaria (11).

La preparación profesional de calidad incluye aspectos éticos, contenidos curriculares acordes con el desarrollo de cada país, con una preparación cónsona con los cambios y procesos tecnológicos aplicados en cada uno de los campos laborales donde deba desempeñarse. Una formación, donde se consideren las posibilidades de campo de empleo y el acceso y necesidades de los profesionales y de las personas a quienes van dirigidos los cuidados de enfermería, en cuanto a preparación académica y número de egresados por año.

Los procesos a través de los cuales ha evolucionado la enfermería, han permitido incrementar el número de personal calificado en materia de conocimientos científicos y prácticos, que permiten el desarrollo cada día de la profesión con aportes teóricos en beneficio del cuidado de calidad a la población.

Los cambios socioeconómicos y tecnológicos, así como los grandes problemas de salud que enfrenta la sociedad, han impulsado la necesidad de contar con enfermeras competentes, de allí que la profesionalización personal de enfermería se constituyó en una prioridad para los países de América Latina, con lo cual, se ha respondido al compromiso en materia de formación a nivel superior, incrementándose la matrícula y los centros donde se imparte esta profesión.

Por otro lado, dado los procesos de continuo cambio, se crea la necesidad de 
una actualización permanente del personal de enfermería, a fin de que sus competencias, respondan a los cambios de perfiles profesionales acordes a las demandas sociales y tecnológicas.

La profesión en su concepto básico está referida con preparación y estudios a nivel superior, preparación para el área laboral por medio de la adquisición de conocimiento, destrezas y habilidades que aporten las herramientas necesarias para un desempeño adecuado ante las diferentes situaciones y vicisitudes que se puedan presentar en el campo laboral, con la creación y ampliación de un cuerpo de teorías sólidas que le permitan a la persona, construir su propio criterio ante determinadas situaciones.

La enfermería es considerada una profesión liberal y una disciplina de carácter social, cuyos sujetos de atención son la persona, la familia y la comunidad, con sus características socioculturales, sus necesidades y derechos, así como el ambiente físico y social que influye en la salud y en el bienestar. Cimienta su práctica en los conocimientos sólidos y actualizados de las ciencias biológicas, sociales y humanísticas y en sus propias teorías y tecnologías. Tiene como fin, dar cuidado integral, ayudar a desarrollar al máximo los potenciales individuales y colectivos, para mantener prácticas de vida saludables, que permitan salvaguardar el estado de salud en todas las etapas de la vida (12).

Tanto la profesión como el proceso de formación que se lleva a cabo dentro de las instituciones de educación superior, están ligados de forma permanente a la evolución de las sociedades, en la estructura social a la que pertenece, enmarcada en un código ético $(13,14)$. En este sentido, involucra el desarrollo del quehacer de forma consciente, comprometida y organizada, tanto individual como grupalmente (15).
La profesionalización se puede ver como el camino por recorrer, desde los más específicos que conllevan a la capacitación teórica y práctica de la persona para su desempeño como profesional en un campo laboral determinado, que debe contar con una preparación acorde y el desempeño de competencias que le permitan ejercer como un profesional.

\section{La competencia profesional}

La competencia es un factor fundamental para la formación profesional, siendo esta la meta primordial, es entendida como el conjunto de saberes (conocimientos), el saber hacer (habilidades) y el saber ser (actitudes y comportamientos), que lleven al profesional a saber manejar su profesión, con todas las cualificaciones y capacidades para resolver situaciones concretas de trabajo (16).

Las competencias constituyen el conjunto de características de una persona, que está directamente relacionado con la buena ejecución en un puesto de trabajo o de una determinada tarea. Las competencias desarrollan en la persona sus capacidades individuales para desenvolverse como un ser integral para la sociedad y para su ejercicio laboral, actuando con conocimientos basados en teorías y evidencias de hechos y experiencias ante acontecimientos similares, respondiendo de manera eficiente (17). Éstas permiten resolver problemas de forma autónoma y creativa, para colaborar en su entorno laboral y en la organización del trabajo (17).

Las competencias es un término incluido de manera persistente en los ámbitos de educación universitaria de enfermería, tanto las genéricas de la profesión como las específicas, para el cuidado de la salud, ya que denotan desarrollo integral y completo en función 
del ser y quehacer, tanto en conocimientos como en su expresión práctica, de funcionalidad para la resolución de situaciones laborales con el mejor uso de los recursos disponibles, en el menor costo y tiempo.

\section{CONCLUSIÓN}

$\mathrm{P}$ ara concluir, la enfermería posee características que la hacen única dentro del campo de la prestación de servicios sanitarios, que requiere condiciones y formación precisa para desempeñarse con arte, disciplina y ciencia que la hacen una profesión única.

Esta profesión conto con los aportes d distintas teorizantes quienes realizaron contribuciones significativas al cuerpo de teorías basados en hechos científicos, sin desligarla de su proceso evolutivo donde se consideró una ocupación basada en conductas condicionadas por un orden lógico que establecía las directrices del cuidado a realizar, un arte ya que es en el cuidado humano y de sus necesidades, donde se desarrolla la práctica como lo describen las teorizantes Ernetine Wiedenbach y Virginia Henderson. Una disciplina para llevar a cabo el cuidado con el mayor orden y esmero en la búsqueda de los resultados más eficaces para la recuperación de la personas, hasta lograr consolidarse como una ciencia y una profesión con un cuerpo teórico propio que hace de enfermería una profesión única, humanitaria, con un gran desarrollo de todas las habilidades y competencias en pro del cuidado del paciente.

Para llegar a ser considerada enfermería como una profesión paso por todo un proceso evolutivo que implico el desarrollo de una fundamentación propia para la justificación e inclusión dentro de las ciencias de la salud y como parte fundamental en la recuperación del paciente y progreso de la relación saludenfermedad.
Estudio apegado a las normas y tratados bioéticos.

- Conflicto de intereses: Ninguno declarado por la autora.

- Financiación: Autofinanciado.

- Agradecimientos: Ninguno declarado por la autora.

\section{REFERENCIAS BIBLIOGRÁFICAS}

1. Amaro $M$, Florence Nightingale, la primera gran teórica de enfermería. Rev Cubana Enfermer [Internet]. 2004 Dic [citado 2018 Mar 07]; 20(3). Disponible en: http://scielo.sld.cu/scielo.php?script= sci_arttext\&pid=S086403192004000300009\&lng=es.

2. Arias P, Mejía-Garzón MA, MatusSánchez D. Revisión documental basada en la teoría de Martha Rogers: cuidado armónico a las adolescentes gestantes en Bogotá en el año 2017. Rev. Iberoam. Educ. Investi. Enferm. 2018; 9(1):45-51.

3. Hernández A, Guardado C. La Enfermería como disciplina profesional holística. Rev Cubana Enfermer [Internet]. 2004. [citado 2018 Mar 06]; 20(2): 11. Disponible en: http://scielo.sld.cu/scielo.php?script= sci_arttext\&pid=S086403192004000200007\&lng=es.

4. Barroso Z, Torres J. Fuentes teóricas de la enfermería profesional: Su influencia en la atención al hombre como ser biopsicosocial. Rev. Cubana Salud Pública [Internet]. 2001. [citado 2018 Mar 06]; 27(1):11-18. Disponible en: http://scielo.sld.cu/scielo.php?script= sci_arttext\&pid=S0864$34662001000100002 \& \operatorname{lng}=\mathrm{es}$.

5. Hernández C. El modelo de Virginia Henderson en la enfermería práctica. Tesis. Universidad de Valladolid. España. 2015. Disponible en: https://uvadoc.uva.es/bitstream/han dle/10324/17711/TFG-

6. Moreira Thereza Maria Magalhães, Araújo Thelma Leite de. 0 modelo conceitual de sistemas abertos 
interatuantes e a teoria de alcance de metas de imogene king. Rev. LatinoAm. Enfermagem [Internet]. 2002 [cited 2018 Mar 07]; 10(1): 97107. Available from: http://www.scielo.br/scielo.php?scri pt=sci_arttext\&pid=S0104-

11692002000100015\&lng=en. https://doi.org/10.1590/S010411692002000100015.

7. González T. Florence Nightingale. Profesionalización de los cuidados desde una perspectiva de la antropología feminista. [Internet] Revista Antropología. 2005; 1(17)

8. Attewell A. Florence Nightingale (1820-1910). Revista Trimestral de Educación Comparada. 2000; 28(1):173-189

9. Roldán R. Modelos de desarrollo y formación de enfermería en la universidad venezolana: Un estudio comparativo. Educere [Internet] 2009 [citado julio 2018]; 13(45):359369.Disponible en: http://ve.scielo.org/scielo.php?script =sci_arttext\&pid=S131649102009000200012\&lng=es\&tlng

10. Arriata A. Investigación y documentación histórica en enfermería. Revista Texto Contexto Enferm. [Internet]. 2005; 14(4):56774. Disponible en: http://www.scielo.br/pdf/tce/v14n4 /a14v14n4

11. Marriner A, Raile M. Modelos y teorías de enfermería. España: Mosby-Doyma; 2003

12. Cuello P. La educación técnica industrial y para el trabajo y las exigencias del mercado laboral. Investigación y postgrado. [Internet]. 2010 [citado 201820 de junio]; 21(1): 11-48. Disponible en: http://ve.scielo.org/scielo.php?script =sci_arttext\&pid=S13160082006000100002\&lng=es\&tlng=es

13. Carrillo E, Arras A. Profesionalización de enfermería: hacia una estrategia de cambio. Revista Tecnociencia. 2012:1; 1,1-8

14. Álvarez D. Enfermería en América Latina: Una mirada al horizonte. Av. enferm. [Internet]. 2015; 33(2):295305. doi: http://dx.doi.org/10.15446/av.enfer m.v33n2.37032

15. Jaimes E. La profesionalización del recurso humano de enfermería y calidad del servicio público de salud. Observatorio Laboral Revista Venezolana. [Internet]. 2009; 2(4): 25$47 . \quad$ Disponible en: http://servicio.bc.uc.edu.ve/faces/rev ista/lainet/lainetv2n4/art1.pdf

16. Salas S, Díaz L, Pérez G. Identificación y diseño de las competencias laborales en el Sistema Nacional de Salud. Educ Med Super [Internet]. 2013 [citado 2018 enero]; 27(1): 92102. Disponible en: http://scielo.sld.cu/scielo.php?script= sci_arttext\&pid=S086421412013000100012\&lng=es

17. González V, González R. Competencias genéricas y formación profesional: Un análisis desde la docencia universitaria. Rev. Iberoam. Educ. [Internet]. 2008; 47; 185-209 